\title{
Evaluation of cybersickness in virtual reality in driving simulator
}

\author{
Ana Agić, Lidija Mandić \\ Faculty of Graphic Arts, University of Zagreb, Croatia
}

\begin{abstract}
Virtual reality (VR) devices are becoming a more popular and widespread tool for learning, gaming and entertainment purposes. One of familiar problems of emerging in VR is side effect known as cybersickness, which can be a nuisance for consumers of VR content. This occurrence can be explained as visual and vestibular conflict. The problem with cybersickness lies within the fact that the body is stationary, but eyes perceive motion in virtual reality, also known as vection. Cybersickness symptoms that often occur include blurred vision, headache, vertigo, upset stomach and other. Aim of this research is to observe changes in cybersickness symptoms in two tested conditions (2D display and VR). In this paper, subjective and objective metric of evaluation regarding cybersickness in VR driving simulation are used. Subjective metric is survey and objective metric is electroencephalogram (EEG). Results of the survey indicate which symptoms of cybersickness are more pronounced during driving in virtual environment compared with classic 2D screen experience. Statistically significant difference was found for 6 variables, which include vertigo, blurred vision and headache. Objective metric showed that highest average beta wave was in VR setting, as well as beta/alpha ratio, which is associated with stress and excitement.
\end{abstract}

Key words: virtual reality, cybersickness, driving simulation, EEG.

\section{Introduction}

Virtual reality (VR) in the last few years became popular for various uses, mainly for gaming, medical treatments for different phobias, architecture, and wide range educational purposes [1]. With technological breakthrough, there is more and more of lowcost VR systems available on the market (such as Oculus Rift, HTC Vive, Samsung Gear, PlayStation VR and other). As VR devices offer different and new experiences, some common side-effects occur [2]. Cybersickness has been proven as one of major problems when associating with virtual environments. Usually, it is a common response to real or perceived movement. In VR systems the terms "motion sickness" and "cybersickness" are frequently used for description of the perceived sensation and they are alike but not the same. Stanney, Kennedy and Drexler in their paper examined differences between motion sickness and cybersickness. They concluded that in latter, Simulator sickness score for disorientation, nausea and oculomotor symptoms were much greater than in motion sickness [3]. These terms have something in common, and that is vection, a term that is described as a visual illusion of self-movement. For example, watching a car moving while watching it from a stationary car can give that impression. Vection is considered to be the origin of cybersickness, and it is caused by sensory mismatch between human eyes and proprioceptive system [4]. There are other theories about cybersickness beside of vection (sensory mismatch theory), such as poison theory, postural instability theory and rest frame theory [5]-[7]. Individual experience in VR can be affected by different factors which include, but are not limited to; factors of a human (age, gender, susceptibility to motion sickness, experience in VR etc.), factors associated with VR system (technical 
specifications, resolution, field of view (FOV) etc.), and factors connected to the content in VR itself [8]. Research papers on this thematic explore cybersickness subjectively by different questionnaires, and one often used is Kennedys Simulator Sickness Questionnaire (SSQ), which was originally intended to examine motion/simulator sickness in flight simulators [9]. It has 16 items, placed into three categories: nausea symptoms, oculomotor symptoms and disorientation symptoms. Some other questionnaires, like Presence Questionnaire (PQ) are more focused on the feeling of presence in VR [10]. When it comes to objective measurement of this unpleasant VR side effect, devices like electrocardiogram (ECG), electroencephalograph (EEG), or galvanic skin response (GSR) are used to measure physiological response and to complement subjective data from questionnaires. EEG is a medical device which is used to measure brain activity and changes which occur. The definition of an EEG wave is any change in the potential difference between the pairs of electrodes in the EEG image [11]. Electric activity of the brain is constant and different in accordance with the different phases of wakefulness. EEG waves are a reflection of huge number of neuron activity in cerebral cortex [12]. The frequency of EEG wave is from $1-50 \mathrm{~Hz}$, and amplitude is from 20 to $200 \mu \mathrm{V}$. They are divided into several types according to the frequency of intensity: alpha $(8-13 \mathrm{~Hz})$, beta $(13-35 \mathrm{~Hz})$, gamma (35$80 \mathrm{~Hz})$, theta $(4-8 \mathrm{~Hz})$ and delta $(0.3-4 \mathrm{~Hz})$ waves.

\section{Related work}

Lin et al., in their paper describe experiment in which they use VR based driving environment (no headset, but projectors which create $360^{\circ}$ environment and a real car), motion sickness questionnaire (MSQ) and EEG as an objective method to evaluate motion sickness [13]. Results show strong 8-10 Hz power increase in parietal and motor areas of the brain at subjects with high motion sickness levels. Liu, Peli and Hwang in their research used multiple devices to objectively measure visually induced motion sickness (VIMS). They measured EEG, blood pressure (BP) and heart rate (HR), in testing environment which was a wide field $\mathrm{VR}$ (no headset, but screens creating $220^{\circ}$ FOV) driving simulator. In their results they state that significant differences were found for EEG in the mean values of TP9Delta, FP1Theta, and TP10Beta, and standard deviations of TP9Alpha and TP10Alpha. For HR and BP no statistical significance was found between non- VIMS and VIMS [14]. Egan et al., also used heart rate monitor and electrodermal activity as an objective quality of experience (QoE) evaluation [15]. Their findings include correlation between EDA and some of the selfreported measures, and also that in VR quality of experience ratings were higher than in $2 \mathrm{D}$ display screen.

\section{Equipment and methodology}

This research examines the differences in subjective and objective metrics in virtual reality compared to $2 \mathrm{D}$ display screen. Objective metric is assessed by using an EEG device which is used to detect and measure brain activity. In this research visual stimuli to each participant through two stages was examined. The visual experience was conducted via car driving simulation. Participants used joystick to navigate, move and interact with the environment in both cases, in 2D (classic environment) and in virtual reality by wearing head mounted display (HMD).

Equipment used for this experiment was a PC equipped with Intel i7 processor, NVidia GeForce 1080 graphic card, 16 GB of RAM memory and 27" display. Virtual reality device was HTC Vive (FOV $110^{\circ}$ and screen resolution of $1200 \times 1080 \mathrm{px}$ per eye), combined with Steam controller for navigation. This controller is suitable for both 2D (classic) gaming and for virtual reality gaming. The EEG measuring device was Muse EEG. On Figure 1 used equipment is depicted.

Participants were 10 volunteers, aged from 28 34 years $(M=29,7)$, from which 6 females and 
4 males. Participants were asked if they have any health issues, so only healthy individuals participated. The experiment was explained to them individually prior to procedure, and they were given the controller to try out the driving mechanism. Participants could terminate the participation in the experiment if they wanted for any reason at any time. Participants who took part in this research had normal or corrected to normal eyesight.

The experiment was conducted as follows; participant was seated, and Muse EEG device was placed and adjusted on his head. After adjusting, an EEG recording was taken in a rested state with eyes closed, in duration of three minutes (baseline brain activity). Secondly, participant was given a Steam controller and drove a car simulation in front of a 2D display and EEG recording was taken during driving. After driving he was given a questionnaire to evaluate his experience. Distance from eyes and screen was adjusted by each participant. Thirdly, HTC Vive device was placed onto EEG device on participant's head and he drove the same car simulation in virtual reality and EEG recording was taken for this third measured state. The simulation runway was one of shorter tracks, $5 \mathrm{~km}$ long. At last, participant was given a questionnaire to evaluate his experience in virtual reality regarding his subjective experience of cybersickness. Questionnaire was an adapted version of widely used Kennedy's cybersickness questionnaire. We adapted his questionnaire in such a way that we excluded some questions which we found unessential (like salivation or burping). Carnegie also changed the original form of Kennedy's SSQ for his research and excluded some questions [16]. In this research questions were as follows: vertigo, blurred vision, difficulty to focus on objects, headache, sweating, stomach nausea, need to vomit and eye strain. For answer evaluation we used Likert type scale from 1 (being least) to 5 (being most). For each participant experiment lasted about 10$15 \mathrm{~min}$ (with included introduction, test drive, measured drive sessions and questionnaires).

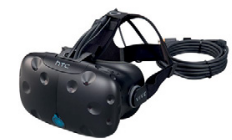

a)

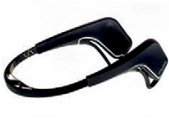

b)

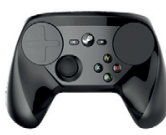

c)
Figure 1: a) HTC Vive b) Muse EEG c) Steam controller

\section{Results and discussion}

This section is dedicated to results acquired from questionnaire and EEG measurements. Results from the questionnaire were obtained with the SPSS statistical program. Since the Shapiro - Wilk test showed that the tested variables deviated significantly from the Gaussian curve, nonparametric statistical methods were used in addition to the basic descriptive statistics. As the two tested situations are being compared in this experiment, the Wilcoxon nonparametric test was used to test the dependent groups of subjects [17]. Statistical difference was defined at $p<0.05$. The results of Wilcoxon matched pairs test are shown in Table 1 and significant results are bolded.

\begin{tabular}{|l|l|l|l|l|l|l|l|l|}
\hline & $\begin{array}{l}\text { Vertigo_ } \\
\text { VR - } \\
\text { Vertigo_2D }\end{array}$ & $\begin{array}{l}\text { Blurred } \\
\text { vision VR } \\
- \text { Blurred } \\
\text { vision 2D }\end{array}$ & $\begin{array}{l}\text { Difficulty } \\
\text { to focus on } \\
\text { objects_VR - } \\
\text { Difficulty } \\
\text { to focus on } \\
\text { objects_2D }\end{array}$ & $\begin{array}{l}\text { Headache_ } \\
\text { VR - } \\
\text { Headache_2D }\end{array}$ & $\begin{array}{l}\text { Sweating_VR } \\
\text { - Sweating_2D }\end{array}$ & $\begin{array}{l}\text { Stomach } \\
\text { nausea_VR } \\
\text { - Stomach } \\
\text { nausea_2D }\end{array}$ & $\begin{array}{l}\text { Need to } \\
\text { vomit._VR } \\
\text { - Need to } \\
\text { vomit._2D }\end{array}$ & $\begin{array}{l}\text { Eye strain_ } \\
\text { VR - Eye } \\
\text { strain_2D }\end{array}$ \\
\hline$Z$ & -2.121 & -2.549 & -2.588 & -2.456 & -1.474 & -2.121 & -1.890 & -2.714 \\
$p$ & $\mathbf{. 0 3 4}$ & $\mathbf{0 . 0 1 1}$ & $\mathbf{0 . 0 1 0}$ & $\mathbf{0 . 0 1 4}$ & 0.140 & $\mathbf{0 . 0 3 4}$ & 0.059 & $\mathbf{0 . 0 0 7}$ \\
\hline
\end{tabular}

Table 1: Significance of the difference between the tested variables in 2D and VR conditions 
From Table 1. above can be observed that six out of eight compared variables showed statistically significant difference. The first compared pair was vertigo in $2 \mathrm{D}$ and $\mathrm{VR}$ conditions where it was observed that subjects felt greater vertigo in VR car driving conditions $(\mathrm{Z}=-2.121, p=0.034)$. Blurred vision is also a variable with a statistically significant difference $(Z=-2.549, p=0.011)$ where vision is more blurred for subjects in a VR environment, as well as difficulty focusing on objects $(\mathrm{Z}=-2.588, p=0.010)$. Headache is more pronounced in VR environment ( $\mathrm{Z}=-2.456, p=0.014)$, as well as stomach nausea $(Z=-2.121, p=0.034)$, while the observed variable need to vomit did not yield statistically significant difference $(Z=-1.890$, $p=0.059)$. The variable eye strain was more pronounced in VR situation, which was the expected result.

Readings from the Muse 2 EEG device were captured by the Mind Monitor application for mobile devices. Readings were afterwards imported into NeuroVisual App, which among other options, depicts the topological image of the brain where brain activity can be seen in relation to the observed situation. Figure 2 depicts all three measured states in brain activity for one participant (where green and orange color in circles present activity in specific band-alpha, beta, delta, theta and gamma). It can be observed that the activity of the brain changes through all three measured states. The neutral state measurement shows the activity of alpha waves in the back of the brain and some beta waves in the front part of the brain. The activity in delta, theta, alpha and beta waves is noticeable in the 2D display testing condition, and even more amplified in the VR testing condition, as can be observed when comparing second and third part of the Figure 2.

Mind Monitor application also calculates the absolute band power (for alpha, beta, delta, theta and gamma band) for each measured state. Absolute band power for a frequency is calculated as the logarithm of the sum of the Power Spectral Density (PSD) of the EEG data for that frequency range, and is measured in

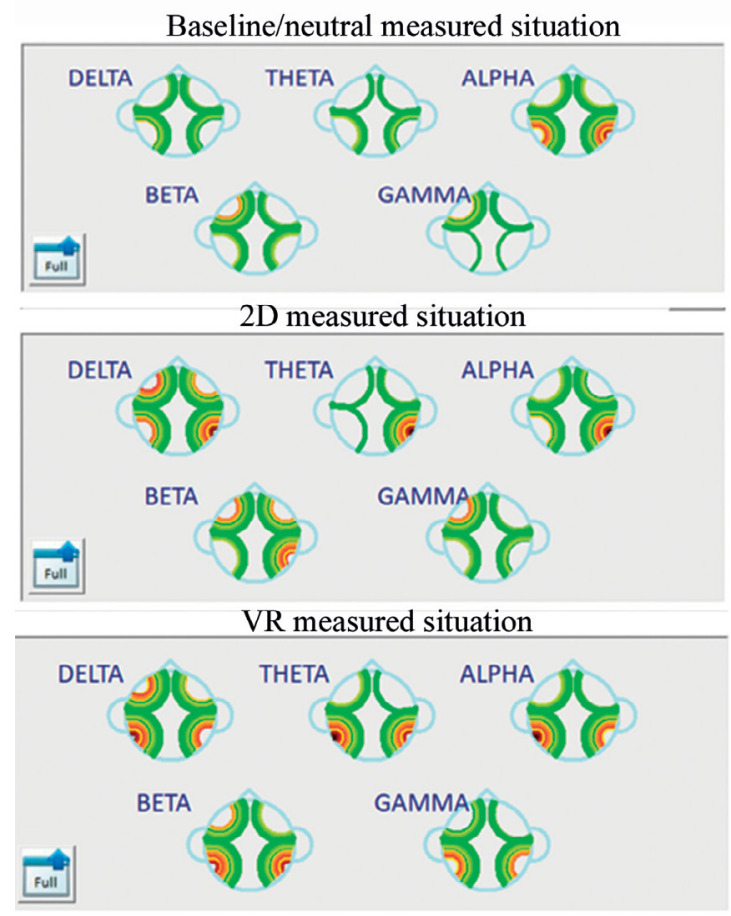

Figure 2. Brain activity across three measured states (for one participant)

decibels $(\mathrm{dB})$ [18]. Figure 3 depicts the average of the beta wave for all participants for all three measured states.

Figure 3 shows that the beta wave (average for all participants) is predominant in the virtual environment compared to driving a car in a $2 \mathrm{D}$ environment, which can be explained with increased attention since there is a higher degree of immersion in the virtual environment than in classical gaming. Also, some authors associate the increased activity

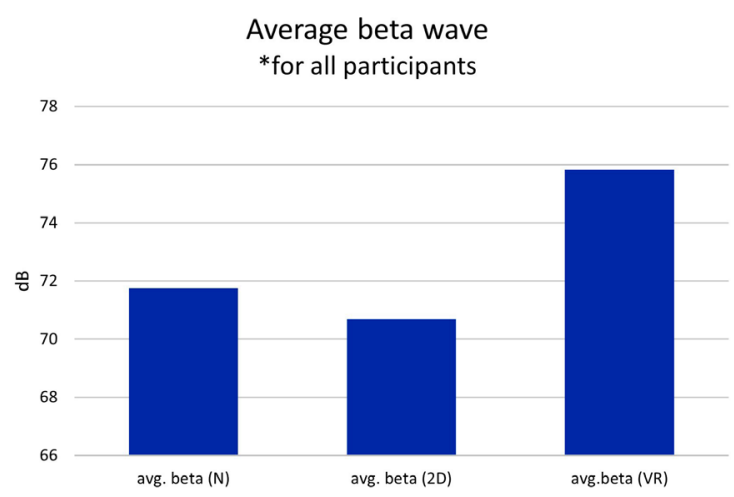

Figure 3. Average beta wave 


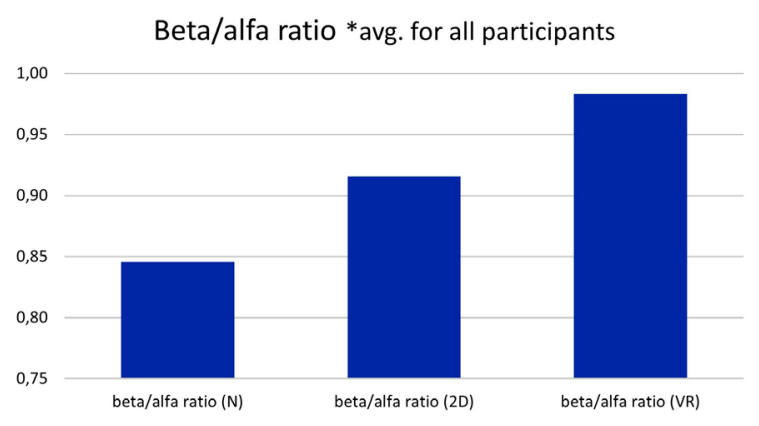

Figure 4: Ratios of average values of beta / alpha waves for all 3 measured states

of beta waves with excitement, which is also called "beta stress" in the literature, which is calculated as the beta/alpha ratio [19][21]. This beta/alpha ratio (average for all participants) is shown in Figure 4 and this result can be explained in such a way that for people who have little or no experience with VR technology, such an experience can be an exciting and/or stressful event.

Also, in Figure 5 below, the theta/beta wave ratio (average for all participants) is calculated for all 3 measured states, where it can be observed that the theta/beta wave ratio is the highest in the neutral state and the lowest in virtual reality while driving (average for all participants). Such a result was obtained by Wen and Aris in their 2020 paper, in which they measured alpha/beta and theta/beta ratios in virtual reality stress research by showing a post-apocalypse scene with zombies in which the respondent rides like in an amusement park [22]. From this result of the theta/beta ratio, it can be concluded that the theta brain wave generally decreases in the examined situation, and the concentration of beta brain waves increases, which is associated with increased concentration and excitement in healthy individuals.

\section{Conclusion}

It has been proven in many previous researches that emerging into virtual reality may cause cybersickness. In this research,

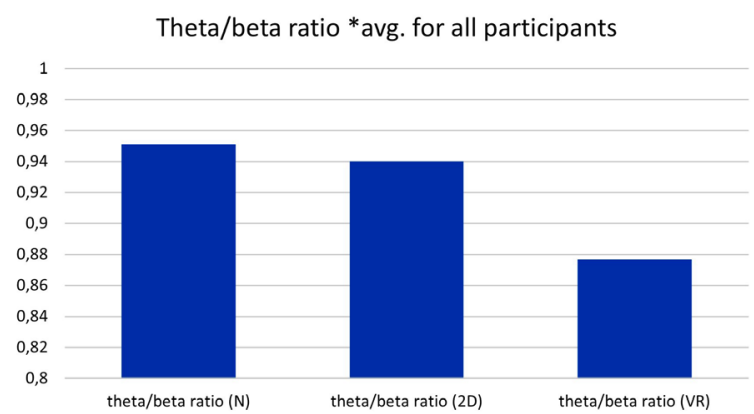

Figure 5. Ratios of average values of theta / beta waves for all 3 measured states

the user experience of car driving simulation in $2 \mathrm{D}$ and VR conditions was investigated. The survey was used as a subjective metric of user experience evaluation and the Muse EEG device for measuring brain activity as an objective metric. The aim of the research was to determine whether there are differences in certain brain waves in different driving conditions, and what is the subjective experience of different experiences in relation to the medium of image display, that is driving in $2 \mathrm{D}$ and VR environment in this case. The results of the objective metric show that the beta/alpha ratio is highest when driving in VR conditions and lowest when in resting (neutral) state. Also, the theta/beta ratio is the lowest in VR conditions and the highest at rest, which is in line with previous research. In the results of the survey, it can be noticed that the more pronounced subjective feeling of discomfort is higher in the six examined variables in VR condition, some of which are dizziness, blurred vision, headache and nausea in the stomach. Some respondents have been observed to have more negative symptoms in VR, although when they completed testing they commented that VR was more fun for them. From this observation, it can be concluded that the habituation process could reduce the negative symptoms that occur. Future work will be oriented towards including more objective metrics like galvanic skin response and heart rate into research of cybersickness and focusing on different contents of VR experiences. 


\section{References}

[1] C. Botella, R. M. Baños, C. Perpiñá, H. Villa, M. Alcañiz, and A. Rey, "Virtual reality treatment of claustrophobia: a case report", Behav. Res. Ther., vol. 36, no. 2, pp. 239-246, Feb. 1998.

[2] S. Palmisano, R. Mursic, and J. Kim, "Vection and cybersickness generated by head-and-display motion in the Oculus Rift," Displays, vol. 46, pp. 1-8, Jan. 2017.

[3] K. M. Stanney, R. S. Kennedy, and J. M. Drexler, "Cybersickness is Not Simulator Sickness," Proc. Hum. Factors Ergon. Soc. Annu. Meet., vol. 41, no. 2, pp. 1138-1142, Oct. 1997.

[4] S. M. LaValle, Virtual reality. Cambridge University press, 2017.

[5] L. Rebenitsch and C. Owen, "Review on cybersickness in applications and visual displays," Virtual Real., vol. 20, no. 2, pp. 101-125, Jun. 2016.

[6] F. Steinicke, Y. Visell, J. Campos, and A. Lécuyer, Human Walking in Virtual Environments. New York, NY: Springer New York, 2013.

[7] A. Tiiro, "Effect of Visual Realism on Cybersickness in Virtual Reality (Master's Thesis)," University of Oulu, 2018.

[8] J. Barrett, "Side effects of virtual environments: A review of the literature - report," Edinburgh South Australia 5111, Austalia, 2004.

[9] R. S. Kennedy, N. E. Lane, K. S. Berbaum, and M. G. Lilienthal, "Simulator Sickness Questionnaire: An Enhanced Method for Quantifying Simulator Sickness," Int. J. Aviat. Psychol., vol. 3, no. 3, pp. 203-220, 1993.

[10] B. G. Witmer and M. J. Singer, "Measuring Presence in Virtual Environments: A Presence Questionnaire," Presence Teleoperators Virtual Environ., vol. 7, no. 3, pp. 225-240, Jun. 1998.

[11] S. Noachtar, C. Binnie, J. Ebersole, F. Mauguière, A. Sakamoto, and B. Westmoreland, "A glossary of terms most commonly used by clinical electroencephalographers and proposal for the report form for the EEG findings," Electroencephalogr. Clin. Neurophysiol. Suppl., vol. 52, pp. 21-41, 1999.

[12] M. Judaš and I. Kostović, Temelji neuroznanosti. Zagreb: Medicinska dokumentacija, 1997.
[13] C.-T. Lin, S.-W. Chuang, Y.-C. Chen, L.-W. Ko, S.F. Liang, and T.-P. Jung, "EEG Effects of Motion Sickness Induced in a Dynamic Virtual Reality Environment," in 2007 29th Annual International Conference of the IEEE Engineering in Medicine and Biology Society, 2007, vol. 2007, no. 2, pp. 38723875.

[14] R. Liu, E. Peli, and A. D. Hwang, "Measuring visually induced motion sickness using wearable devices," Electron. Imaging, vol. 2017, no. 14, pp. 218-223, Jan. 2017.

[15] D. Egan, S. Brennan, J. Barrett, Y. Qiao, C. Timmerer, and N. Murray, "An evaluation of Heart Rate and ElectroDermal Activity as an objective QoE evaluation method for immersive virtual reality environments," in 2016 Eighth International Conference on Quality of Multimedia Experience (QoMEX), 2016, pp. 1-6.

[16] K. Carnegie, "Mitigating Visual Discomfort on Head Mounted Displays using Estimated Gaze Dependent Depth of Field, Master Thesis," Victoria University of Wellington, 2015.

[17] M. Marusteri and V. Bacarea, “Comparing groups for statistical differences: how to choose the right statistical test?" Biochem. Medica, vol. 20, no. 1, pp. 15-32, 2010.

[18] Muse, "Absolute Band Powers." [Online]. Available: https://web.archive.org/web/20181105231756/ http://developer.choosemuse.com/tools/availabledata\#Absolute_Band_Powers. [Accessed: 15-Sep2020].

[19] A. Riera et al., "Electro-physiological data fusion for stress detection," in Studies in health technology and informatics, 2012, vol. 10, no. September, pp. 228232.

[20] S.-H. Seo and J.-T. Lee, "Stress and EEG," in Convergence and Hybrid Information Technologies, no. March, InTech, 2010.

[21] D. Plass-Oude Bos, "EEG-based Emotion Recognition," Influ. Vis. Audit. Stimuli, 2006.

[22] T. Y. Wen and S. A. M. Aris, "Electroencephalogram (EEG) stress analysis on alpha/beta ratio and theta/ beta ratio," Indones. J. Electr. Eng. Comput. Sci., vol. 17, no. 1, pp. 175-182, 2020. 